\title{
Optimum plasmon hybridization in percolation threshold of silver films near metallic surfaces
}

\author{
A. I. Maaroof and D. S. Sutherland* \\ iNANO Center, Ny Munkegade 118, Building 1521, University of Aarhus, Aarhus 8000,Denmark \\ * Corresponding author. E-mail: duncan@inano.dk.
}

\begin{abstract}
We demonstrate experimentally a strong interaction and, plasmonic hybridization from a nanosystem having both localized and delocalized surface plasmons modes simultaneously in the presence of a nearby thin continuous metallic film. In situ DC resistance measurement of silver films and percolation theory was used to determine accurately the systems where the percolation threshold exists by distinguishing the nucleation and growth regions of silver films. We found an optimum plasmon hybridization existing in this percolation threshold region which can be verified from the absorption spectra. We interpret our data in terms of a fitting of the absorption spectra to the Fano type line shapes model.
\end{abstract}

\section{Introduction}

Recently there has been huge interest in studying various metallic nanostructures capable of manipulating light in order to obtain unique optical properties. These properties are explained by their plasmon resonances behavior and strongly depend on the geometry, material and surrounding environment. They can be easily manipulated to suit different applications ranging from chemical and biological sensors [1-3], medical [4,5], waveguiding [6-8] and to metamaterials [9-12]. Most of these already obtained nanostructures suffer from losses and are not highly intensified which makes their applications limited or less efficient. However, extremely strong and localized electromagnetic fields generated in some of these metallic nanostructures have been achieved by using plasmonic interactions phenomena normally between two well known plasmonic modes, namely, localized surface Plasmon (LSP) which can represented by the subwavelength nanostructures and delocalized or surface plasmon polariton (SPP) which can represented by the continuous metallic films in both theoretically [13-18] and experimentally [19-21].

In this paper we show that plasmonic interactions can also be enhanced, not only very strongly, but reach optimum for a nanosystem having both modes simultaneously in the presence of a nearby thin continuous metallic film, compared with localized surface plasmon surfaces interactions. The coexistence of localized and delocalized surface plasmon modes at the percolation threshold has been proven theoretically [22] and experimentally [23], however it remains a challenge to find such percolation threshold conditions experimentally.

\section{Experimental Details}

\subsection{Samples preparation}

2.1.1. Percolation samples. Single-layer thin films of Ag were deposited on super white glass substrates using high vacuum, DC magnetron sputtering. The sputtering target was a 99.999\% Ag disc (50mm diameter), placed $150 \mathrm{~mm}$ away from the substrate. The substrate was rotated at $10 \mathrm{rpm}$ and placed at $150 \mathrm{~mm}$ to the flux of silver. The base pressure was better than $\cong 10^{-6}$ Torr, while sputtering was carried out in presence of flowing Ar, at a pressure of $\sim 2$ mTorr. The results are shown in figure 1. The oxidation of the prepared thin silver films on exposure to air was mitigated by measuring immediately after samples were fabricated. The oxidation of silver represents a significant challenge to application of percolating silver films, however capping of silver films with barrier oxides or alternate plasmonic materials such as gold may represent potential application materials. 
2.1.2. Plasmon hybridization samples: The geometry of nanosystems used is shown as inset graph in Fig.2 and consists of a three layers structure deposited on glass substrate. First, continuous silver film of thickness $40 \mathrm{~nm}$ was sputtered onto a glass substrate and alumina (A12O3) as spacer layer of thickness 40nm was deposited over the silver surface and finally the required silve $\mathrm{r}$ film region was deposited on top of the spacer with precise measurement of the mass thickness by quartz-crystal film-thickness monitor. The Silver and Alumina layers were prepared by using high vacuum DC and RF high vacuum magnetron sputtering system, respectively. The sputtering targets of $\mathrm{Au}$ and $\mathrm{Al} 2 \mathrm{O} 3$ were $99.99 \%$ pure discs, placed $70 \mathrm{~mm}$ away from the substrate. The base pressure was better than $\sim 10^{-7}$ Torr, while sputtering was carried out in the presence of flowing $\mathrm{Ar}$, at a pressure of $\sim 2$ mTorr.

\subsection{Characterization}

2.2.1. Microscopy. FEI Nova-600 FEG NanoSEM Scanning electron microscopy (SEM) was used to study the nanostructure and cross-sections of the films. The Nova NanoSEM features a helix detector that delivers high resolution images in low vacuum as well as at low $\mathrm{kV}$.

2.2.2. Spectrophotometry. Spectral transmittance $T(\lambda)$ and reflectance $R(\lambda)$ for all the samples were measured by using Shimadzu UV-3600 UV-VIS-NIR Spectrophotometer over the visible and NIR wavelength ranges 300nm $\leq \lambda \leq 2000 \mathrm{~nm}$. Reflectance was measured at the near normal $\theta=8^{\circ}$ and the absorbance $A(\lambda)$ is deduced by using $A(\lambda)=1-R(\lambda)-T(\lambda)$.

\section{Results and discussions}

In this experiment, the percolation threshold region has been traced and determined accurately by studying the nucleation and growth of silver films on glass substrate using in situ DC resistance measurement deposited by DC magnetron sputtering and percolation theory. The main growth stages are (see figure 1) with increasing deposited material or the fractional coverage $(f)$; the initial nanoislands stage $\left(f \leq f_{c}\right)$, where $f_{c}$ is the fractional coverage at percolation threshold, then the percolation region $\left(\left(f=f_{c}\right)\right.$, our main focus), and the semicontinuous region $\left(f \geq f_{c}\right)$ until the film becomes continuous $(f=1)$. This procedure is similar to the one described by Maaroof and Evans [24], accordingly, the experimental value of the percolation threshold $\left(f_{c}\right)$ of silver films has been estimated by using the equation $R\left(f_{c}\right)=\left(R_{D} R_{M}\right)^{1 / 2}$, where $\mathrm{R}_{\mathrm{D}}$ is the dielectric resistance value at $f=0$ and $\mathrm{R}_{\mathrm{M}}$ is the metallic resistance value at $f=1$, hence $f_{c}$ corresponding to the $R\left(f_{c}\right)$ is found (from the measured values see Ref. [24] for more details). Thus, it was found that the value of critical fractional coverage is $f_{c}=0.45$ (with equivalent mass thickness $=4 \mathrm{~nm}$ ). To add more accuracy, various samples have been prepared and characterized around this region both optically and structurally to confirm this. 


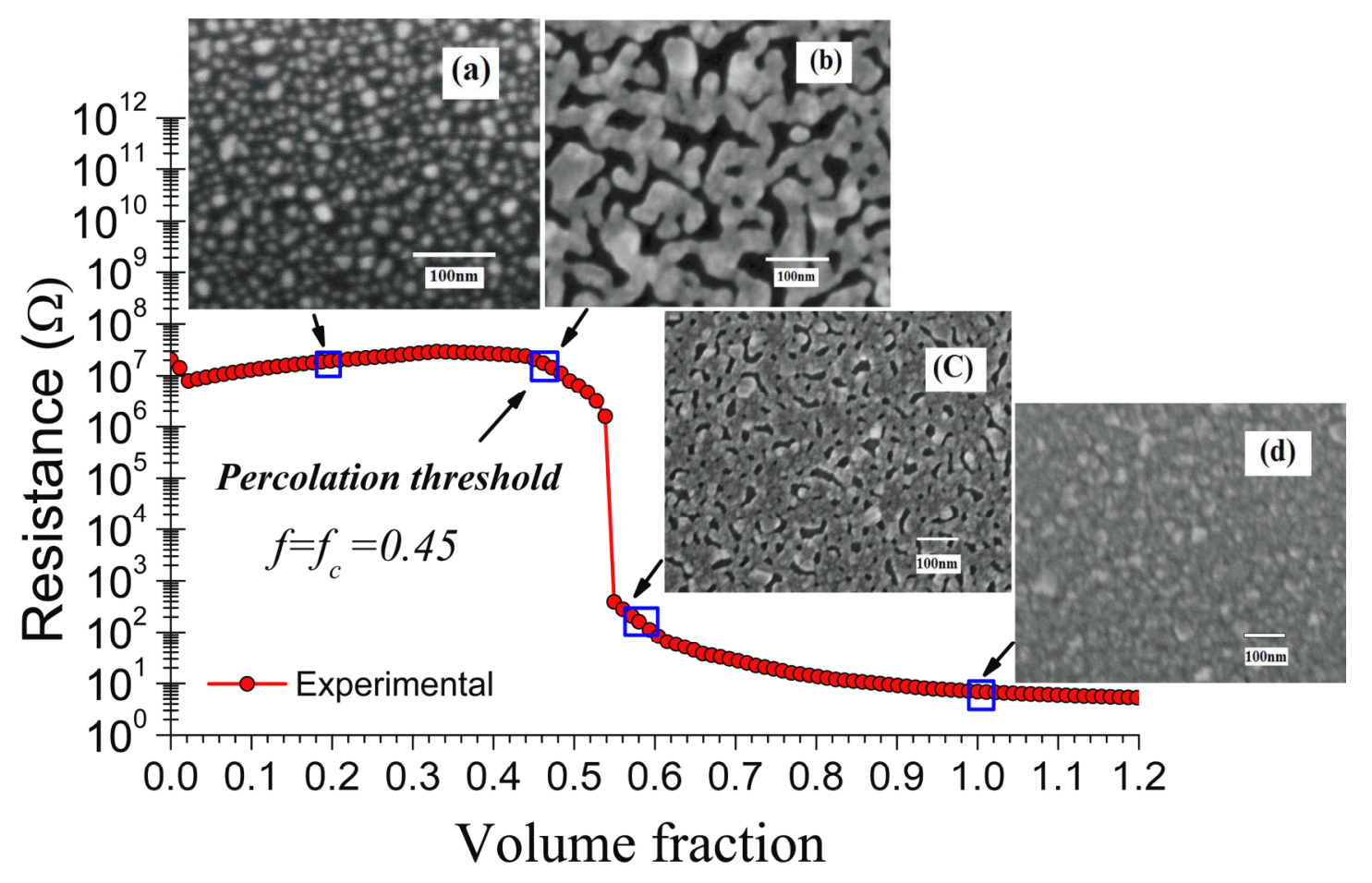

Figure 1. DC film resistance measurement of a growing silver film on glass substrate as a function of volume fraction $(f)$. The four inset graphs showing SEM images of silver growth processes. These are (a) the initial nanoislands stage $\left(f \leq f_{c}\right)$ with mass thickness $2 \mathrm{~nm}$, (b) the percolation threshold region $\left(\left(f=f_{c}\right)\right.$ with mass thickness $4 \mathrm{~nm}$, (c) the semicontinuous region $\left(f \geq f_{c}\right)$ with mass thickness $=5 \mathrm{~nm}$ and (d) the film becomes continuous at $(f=l)$ with mass thickness $=9 \mathrm{~nm}$.

When the light strikes one of the surfaces mentioned above, it generates electron waves, called plasmons. These surface waves only exist in metals and oscillate naturally to generate local electric fields. Figure 1 shows that there are three types of plasmonic modes depending on the structure of silver films. These are (i) discontinuous structure or islands region with Localized Surface Plasmon (LSP) mode, (see inset image of figure 1 (a)). This mode is confined to the surface and becomes especially strong if the frequency of the excited light approaches the frequencies of plasmon resonances, optically see figure 2 ( $2 \mathrm{~nm} \mathrm{Ag} / \mathrm{glass})$. (ii) The Percolation threshold region with coexistence of localized and delocalized surface plasmon mode (CLDSP), structurally see inset image of figure 1(b) while optically figure 3 ( $4 \mathrm{~nm} /$ glass) (iii) Continuous region with delocalized surface Plasmon (DSP) mode, see figure 1(d). This mode is a surface-plasmon polariton (SPP) or a surface electromagnetic wave propagating along the interface normally between two media possessing permittivities with opposite signs, such as the metal-dielectric interface. 


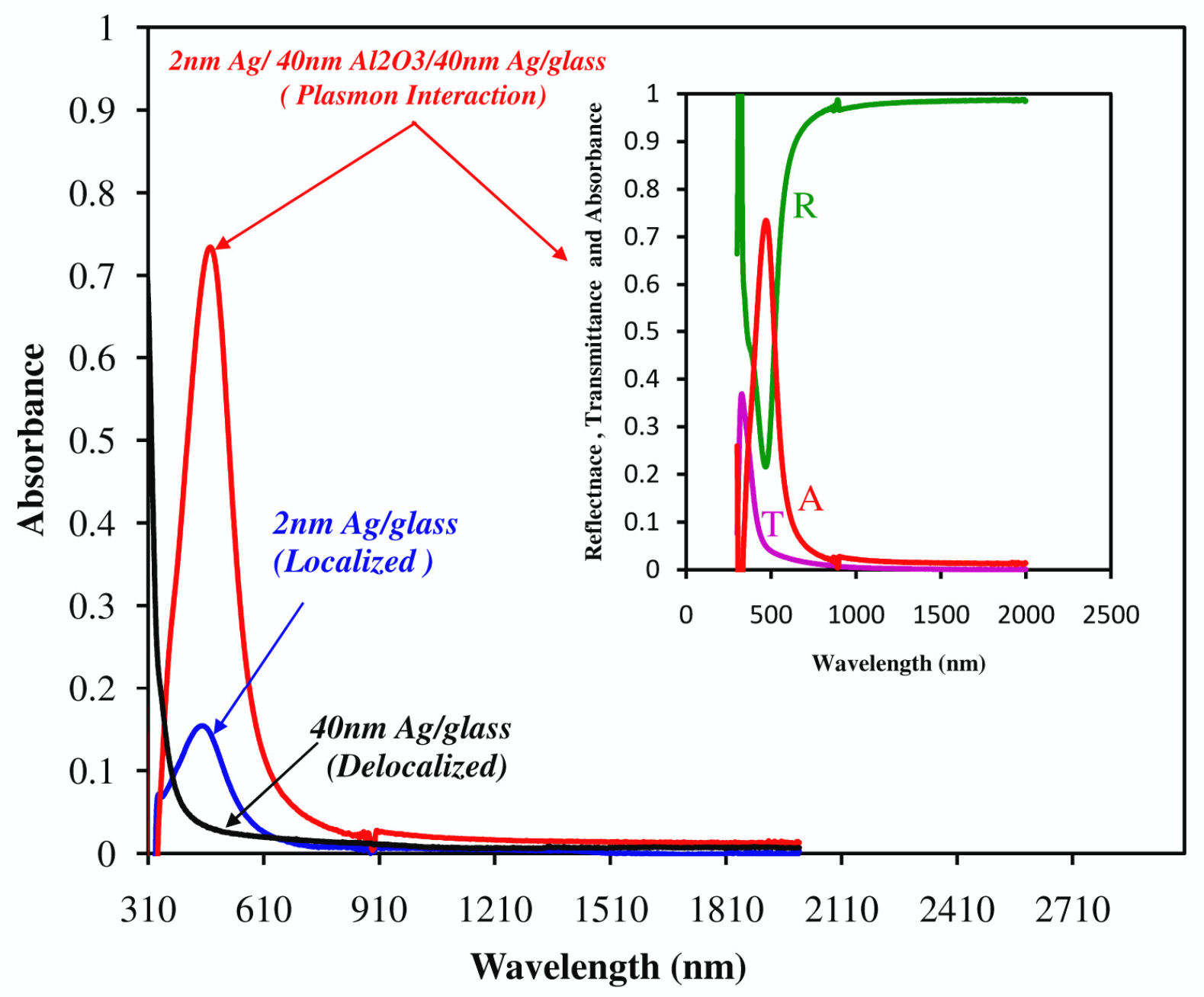

Figure 2. Absorbance spectra as a function of wavelength for a three layers nanosystem used in this experiment in case of nanoislands. The continuous film has a thickness of $40 \mathrm{~nm}$ and the spacer layer $\left(\mathrm{Al}_{2} \mathrm{O}_{3}\right)$ has a thickness of $40 \mathrm{~nm}$ and the top layer has nanoislands film with a mass thickness of $2 \mathrm{~nm}$. In comparison, the measured absorbance for each silver layer has been plotted as well. The inset graph shows the reflectance, transmittance, and absorbance only for three layers nanosystem. 


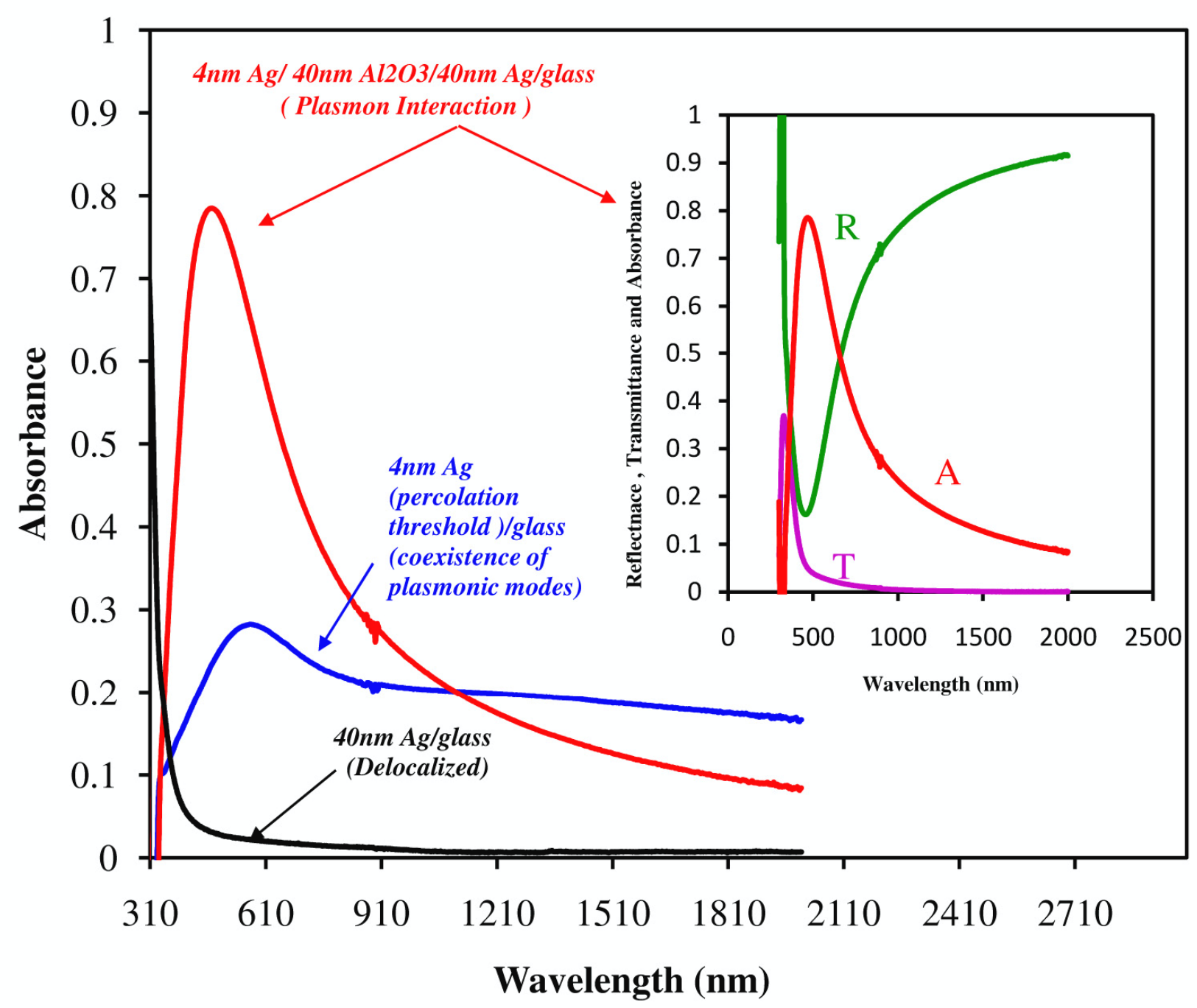

Figure 3. Absorbance spectra as a function of wavelength for a three layers nanosystem used in this experiment in case of percolation threshold. (a) The continuous film has a thickness of $40 \mathrm{~nm}$ and the spacer layer $\left(\mathrm{Al}_{2} \mathrm{O}_{3}\right)$ has a thickness of $40 \mathrm{~nm}$ and the top layer at percolation threshold of silver film has a mass thickness of $4 \mathrm{~nm}$. In comparison, the measured absorbance for each silver layer has been plotted as well. The inset graph shows the reflectance, transmittance, and absorbance only for three layers nanosystem.

To demonstrate strong plasmonic interactions of CLDSP with continuous thins films, we first characterized optically each single layer of the nanosystem as shown in figure 2 and 3 namely, the single layer of silver film $(40 \mathrm{~nm})$ on glass, and compare to the top layer and with the whole nanosystem, figure 2 and 3 show absorption spectra for such a comparison. In figure 2 the top layer is a nanoisland film which represents LSP and figure 3 the top layer is at percolation threshold which represents CDLSP. The single layer of nanoislands with a mass thickness $=2 \mathrm{~nm}$ on glass substrates display the familiar $30 \%$ anomalous absorption at $\lambda_{\mathrm{p}}=472 \mathrm{~nm}$ which is typical for localized plasmon resonances. It is clear that local fields amplified by interaction of the island resonances with 
the incident radiation field while the single layer at the percolating threshold with equivalent mass thickness $\cong 4$ nm exhibit no such pronounce peak, see figure 3 , because the duality of plasmonic behavior on delocalized modes which allow transfer of the resonance over the entire system, and localized modes allow concentration of the resonance in a small part of it [22]. When the nanosystem is formed a significantly enhanced absorption is seen. First, in case where the top layer is composed of nanoislands with $2 \mathrm{~nm}$ mass thickness with mostly localized surface plasmon modes the absorption reaches $73 \%$ and there is no shift in the wavelength as reported in reference [25]. While in case where the top layer is at the percolation threshold, with a $4 \mathrm{~nm}$ mass thickness, the absorption is optimized to nearly 805 at $\lambda_{\mathrm{p}}=485 \mathrm{~nm}$ and this is also clearly shown from the reflectance spectrum as a sharp dip see the inset graph of figure 3. This maximum in the absorption for such nanosystems can confirm two important physical aspects: Firstly, the experimental evidence of the coexistence of localized and delocalized surface plasmons at the percolation threshold. Secondly, the preparation of films at or close to the percolation threshold can provide an addition method of control of plasmonic interactions.

To add more evidence to the reported value of the percolation threshold $\left(f_{c}\right)=0.45$ and the absorption optimization, another value of film thickness has been chosen and studied, just below the percolation threshold, with a mass thickness equal to $4.5 \mathrm{~nm}(f=0.5)$. The SEM image for this region is shown in figure $1(\mathrm{c})$ and the absorption spectrum is plotted together with $2 \mathrm{~nm}, 4 \mathrm{~nm}$ and $4.5 \mathrm{~nm}$ films for comparison as shown in figure 4(a). It is clear that plasmonic interaction just above percolation threshold $(\mathrm{t}=4.5 \mathrm{~nm})$ is also strong and shifted back to a shorter wavelength $\lambda_{\mathrm{p}}=457 \mathrm{~nm}$ but with lower amplitude. That means the film is still percolating but the concentration of the localized surface plasmon resonance on the surface is less than at the percolation threshold region.

We fit the data with a Fano line shape which can be used to explain the interaction of a continuous and discrete state. In the far field, the absorption spectra $A(\omega)=|a(\omega)|^{2}$ can be described by the following model $[26,27,28]$

$$
a(\omega)=a_{r}+\sum_{j=s, a} \frac{-b_{j} \Gamma_{j} e^{i \phi,}}{\hbar \omega-E_{j}+i \Gamma_{j}},
$$

Where $a_{r}$ is the amplitude of a constant background, $b_{j}$ and $\Phi_{j}$ are the amplitude and phase of surface plasmon modes, while $E_{j}$ and $\Gamma_{j}$ represent the spectral position and the linewidth of the individual resonances, and $s$ stands for symmetric and asymmetric modes, respectively. Figure 4(b) shows the absorption spectra fitting for the three interaction experimental cases shown in figure 4(a) below, at and above percolation threshold, $2 \mathrm{~nm}, 4 \mathrm{~nm}$, $4.5 \mathrm{~nm}$, respectively, where the fitting for each case includes only a single resonance. In all three cases, the absorption spectra are dominated by highly symmetric localized surface plasmon peak which can be fitted extremely well by Fano line shapes equation (1), which describes the interaction between localized (discrete state) and delocalized surface Plasmon (continuum state) modes. The curves shown in figure 4(b) are typical symmetric Fano resonance shape profile and shown clearly when they plotted as a function of energy, see inset graph of figure 4(b). The analysis gives insight into potential explanations for the observed optical resonances. The fitting parameters of all above mentioned Fano type absorption spectra are given in the caption of figure 4. 

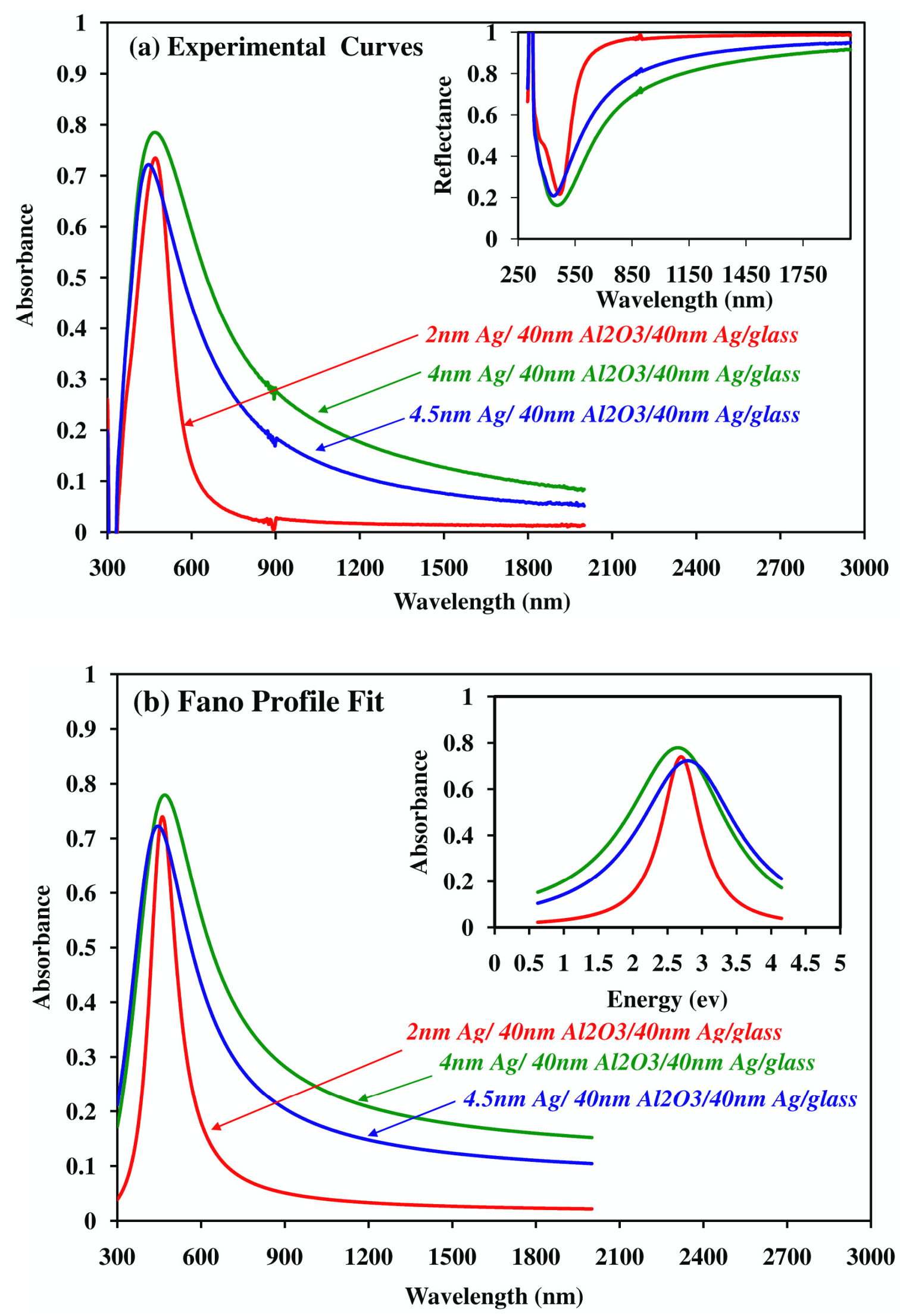
Figure 4. (a) Absorbance spectra as a function of wavelength for three different nanosystems with the top layer thickness vary from 2,4 , and $4.5 \mathrm{~nm}$, below, at, and above percolation threshold, respectively. The inset graph shows the equivalent reflectance for each nanosystem. (b) Compared model simulations of absorbance spectra for the three nanosystems mentioned in (a). The parameters for the nanosystem with the top layer below percolation threshold ( $2 \mathrm{~nm}$ mass thickness ) are $\mathrm{a}_{\mathrm{r}}=0.05, \mathrm{~b}=0.86, \Phi=0, \mathrm{E}=2.7$ and $\Gamma=0.35$; with the top layer at percolation threshold (4nm mass thickness ) are $\mathrm{a}_{\mathrm{r}}=0.05, \mathrm{~b}=0.88, \Phi=0, \mathrm{E}=2.7$ and $\Gamma=0.88$; and with the top layer above percolation threshold ( $4.5 \mathrm{~nm}$ mass thickness) are $\mathrm{a}_{\mathrm{r}}=0.05, \mathrm{~b}=0.85, \Phi=0, \mathrm{E}=2.8$ and $\Gamma=0.88$. The inset graph shows the absorbance plotted as a function of energy to show the resonances as a symmetric.

The inset graph shows the absorbance plotted as a function of energy to show the resonances as a symmetric. That we observe single resonances corresponding to symmetric modes suggests that the asymmetric modes are too weak to show. The exact details of the interaction between the 40nm silver films and the CDLSP are not clear, however the thickness of the metal silver has previously been shown to have critical importance in the coupling between LSPR's and SPP's and interpret in terms of a Anderssen-Fano model [29]. We interpret the system as resulting from the coupled state of the CDLSP interacting with the SPP modes of the thick metal film. The strong coupling that we observe we interpret as an interaction between a coupled state of the continuous and discrete plasmons in the thin film with the SPP in the thick film. We envisage a coupling between the oscillating fields induced by the propagating plasmon at the surface of the thick silver film and that propagating in the thin silver film. The presence of the localised surface plasmon resonances acts both to couple light into the delocalised plasmon modes of the thin film and to the SPP modes in the thick film, but also to modify the energy of the delocalised state in the thin film apparently to aid coupling between this delocalised state and the SPP mode.

\section{Conclusion}

In summary, we have demonstrated the first experimental evidence of optimum plasmonic hybridization at optical frequency in a nanosystem consisting of dual plasmonic modes. We have shown that this unique optical response as a result of plasmonic interactions between the coexistence of localized and delocalized surface plasmon modes which only exist at the percolation threshold of silver films. The result is then compared with localized surface plasmon interactions for more clarification. The spectra are dominated by a highly symmetric localized surface plasmon peak that can be fitted extremely well by the classical Fano equation in term of absorbance, which describes the interaction between localized and delocalized surface plasmons such unique plasmonic properties favorable and open new platform for a range of potential applications using such interesting region, such as sensors, medical, filters, metamaterials and other plasmonic devices especially if we consider using more complicated nanostructures [30].

\section{Acknowledgments}

Some of this work has been prepared with the University of Technology Sydney and is gratefully acknowledged.

\section{References}

[1] Kabashin, A.V.; Evans, P.; Pastkovsky S.; Hendren,W.; Wurtz,G.A.; Atkinson,R.; Pollard,R.; Podolskiy, V.A.; Zayats,A.V.; Nat. Mater. 2009 , 8, 867.

[2] Anker, J.N.; Hall, W.P.; Lyandres, O.; Shah, N.C.; Zhao, J.; Van Duyne, R.P. Nat.

Mater. 2008,7, 442-453.

[3] Stewart,M.E.; Anderton C. R.; Thompson, L. B.; Maria J.; Gray S. K.; Rogers, J. A.

Nuzzo, R.G. Chem. Rev. 2008, 108, 494-521. 
[4] Lal,S.; Link S.; Halas,N.J.; Nat. Phot. 2007, 1, 641-648.

[5] Lal,S.; Clare, S.E.; and Halas, N. J. Acc. Chem. Res. 2008, 41(12) 1842-1851.

[6] Maier S.A.; Kik, P.G.; Atwater, H.A.; Meltzer, S.; Harel, E.; Koel, B.E.; Requicha, A.G.; Nat. Mater. 2003, 2, 229-232.

[7] Cai, W.; Sainidou,R.; Xu,J.; Polman,A.; Javier Garc1'a de Abajo,F.; Nano Lett. 2009, 9, (3),1176-1181.

[8] Oulton, R. F.; Sorger, V. J.; Genov, D. A.; Pile, D. F. P.; Zhang, X. Nat. Photonics 2008, 2, 496-500.

[9] Zhang,S.; Fan,W.; Frauenglass,A.; Minhas,B.; Malloy K.J.; Brueck, S.R.J. Phys. Rev. Lett. 2005, 94, 037402.

[10] Kawata,S.; Ono,A.; Verma, P. Nat. Phot., 2008, doi:10.1038/nphoton,103.

[11] Li,T.; Li,J.; Wang, F.; Wang,Q.; Liu,H.; Zhu, S.; Zhu,Y. Appl. Phys. Lett. 2007,90, 251112.

[12] Henzie,J.; LEE, M.H.; ODOM,T.W.Nat. Nano. 2007, 2, 549-554.

[13] Papanikolaou,N.; Phys. Rev. B 2007,75, 235426.

[14] Khlebtsov,B.N.; Khanadeyev, V.A.; Ye,J.; Mackowski, D.W.; Borghs,G.; Khlebtsov, N.G. Phys Rev. B 2008, 77, 035440.

[15] Christ,A.; T. Zentgraf, T.; Tikhodeev, S.G.; Gippius, N.A.; Martin,O.J.F.; Kuhl,J.; Giessen, H. phys. stat. sol. (b) 2006, 243, No. 10. 2344-2348.

[16] Leveque G.; and Martin, O.J.F. Opt. Exp.2006,14, 21, 9971-9981.

[17] Hao, F.; Nordlander, P.; Sonnefraud,Y.; Van Dorpe, P.; Maier, S.A. 2009, DOI: $10.1021 / n n 900012 r$.

[18] Hao, F., Nordlander, P. Appl. Phy Lett. 2006, 89, 103101.

[19] Kanté,B.; Burokur,S.N.; Sellier,A.; De Lustrac, A.; Lourtioz, J.M. Phys Rev. B 2009,79, 075121.

[20] Pinedo Rivera,T.; Lecarme, O.; Hartmann, J.; Inglebert,R.L.; Peyrade D. Microelectronic Engineering , 2009,86, 1089-1092.

[21] Cesario, J.; Quidant,R.; Badenes G.; Enoch,S. Opt. Lett. 2005, Vol. 30, No. 24

[22] Stockman, M.I.; Faleev,S.V.; Bergman,D.J. Phys. Rev. Lett. 2001,87,167401.

[23] Seal, K.; Genov,D.A.; Sarychev,A.K.; Noh,H.; Shalaev,V.M.; Ying, Z.C.; Zhang,X.; Cao, H. Phys. Rev. Lett. 2006, 97, 206103.

[24] A. I. Maaroof, A.I.; Evans, B.L. J. Appl. Phys. 1996, 76, 1047.

[25] Holland,W.R.; Hall,D.G. Phys. Rev. Lett. 1984, 52,1041.

[26] Miroshnichenko, A.E.; Flach,S.; Kivshar,Y.S. 2009, arXiv:0902.3014v4.

[27] Christ,A.; Ekinci,Y.; Solak, H.H.; Gippius,N.A.; Tikhodeev,S.G.; Martin,O.J.F. Phy. Rev. B 2007, 76, 201405(R).

[28] Hao, F.; Nordlander,P.; Sonnefraud,Y; Van Dorpe, P.; Maier,S.A. AcsNano, 2009, 3, 3, 643-652.

[29] Le, F., Lwin N.Z., Steele J.M., Kall M., Halas N.J.Nordlander P Nano Letters 2005 5,10, 2009

[30] Smith,G.B.; Maaroof,A.I.; Cortie,M.B. Phys Rev. B 2008, 78, 165418. 